\title{
Clinical Presentations and Outcomes of Coronavirus Disease 2019 in Patients With Solid Tumors
}

Imran Farooque ${ }^{1}$, Umar Farooque ${ }^{2}$, Sundas Karimi ${ }^{3}$, Muhammad Usman Shah Syed ${ }^{4}$, Zubia Nadeem ${ }^{5}$, Arif Zulfiqar ${ }^{6}$, Sufyan Mustafa ${ }^{7}$, Rizwan Farooque ${ }^{8}$, Ayyaz A. Sultan ${ }^{9}$, Syed Adeel Hassan ${ }^{10}$

1. Public Health, Peoples University of Medical and Health Sciences for Women, Nawabshah, PAK 2. Neurology, Dow University of Health Sciences, Karachi, PAK 3. Orthopedic Surgery, Dow University Hospital, Karachi, PAK 4. Internal Medicine, Dow University of Health Sciences, Karachi, PAK 5. Medicine, Dow University of Health Sciences, Karachi, PAK 6. Medicine and Surgery, Dow Medical College, Karachi, PAK 7. Medicine, Dow Medical College, Civil Hospital, Karachi, PAK 8. Internal Medicine, Sindh Medical College, Karachi, PAK 9. Hematology/Oncology, California Cancer Associates for Research \& Excellence, Fresno, USA 10. Internal Medicine, University of Louisville, Louisville, USA

Corresponding author: Umar Farooque, umarfarooque65@gmail.com

\section{Abstract}

\section{Background}

Coronavirus disease 2019 (COVID-19) is a global health crisis. The literature suggests that cancer patients are more prone to be affected by COVID-19 because cancer suppresses the immune system and such patients usually present poor results. The objective of this study is to present all clinical, laboratory, and demographic characteristics of COVID-19 patients with solid tumors.

\section{Methodology}

This study was conducted at the Dow University of Health Sciences for a period of six months from April 2020 to September 2020. In this study, we included a total of 1,519 confirmed patients diagnosed with solid tumors via polymerase chain reaction. The mortality timeline within 30 days of contracting the virus was considered, and the median age of the included individuals was 61 years, with a range of $20-95$ years. Of the patients included in the study, $49.4 \%$ (750) were men; moreover, $3.15 \%$ of our study population had prostate cancer, $10.20 \%$ had colorectal cancer, $2.76 \%$ had breast cancer, and $10.46 \%$ had lung cancer. Of the patients, $25.93 \%$ presented with at least one comorbidity. For $73 \%$ of the patients, at least one direct therapy for COVID-19 was included in the treatment; $56.6 \%$ of the patients were hospitalized, and $11.32 \%$ were admitted to the intensive care unit.

\section{Results}

The mortality rate was $4.74 \%$ in the first 30 days after diagnosis, where 72 patients died. The findings of the first multi-variation model showed that males at older ages who were diabetic and going through cytotoxic therapy were prone to die within the first 30 days. However, the 30-day mortality rate was lower in patients diagnosed with prostate and breast cancer. The second set incorporated laboratory factors, where we found

Review began 05/28/2021 Review ended 06/01/2021 Published 06/05/2021

\section{() Copyright 2021}

Farooque et al. This is an open access article distributed under the terms of the Creative Commons Attribution License CC-BY 4.0, which permits unrestricted use, distribution, and reproduction in any medium, provided the original author and source are credited. that higher values of leukocytosis, thrombocytopenia, and lymphocytopenia were correlated with higher rates of mortality within 30 days.

\section{Conclusions}

We conclude that there is a higher mortality rate of COVID-19 in patients with solid tumors than in the general population. However, it was found to be lower in the Pakistani population compared with the Chinese and Western populations. Intensive care can decrease mortality rates in COVID-19 and cancer patients.

Categories: Internal Medicine, Infectious Disease, Oncology

Keywords: pandemic, covid-19, solid tumor, prostate cancer, colorectal cancer, breast cancer, lung cancer, clinical presentation, outcomes, mortality

\section{Introduction}

Coronavirus disease 2019 (COVID-19) is a recent pandemic that has turned out to be a global health crisis. It is a severe acute respiratory syndrome that was reported for the first time in Wuhan, China, in December 2019 and shortly after spread around the globe [1]. Most reports of COVID-19 suggest that this virus affects patients differently according to age and comorbidities. Specifically, it affects people who are older and have comorbidities that are associated with higher risks of mortality and infection. Cancer is a high-risk disease in which patients are at a greater risk because of such vulnerabilities as immunodeficiency. Cancer suppresses patients' immune systems, especially when they are older and have frequent diseases. Early 
reports on COVID-19 suggest that cancer patients are prone to be affected by COVID-19, and such patients usually present poor results [2-8]. As COVID-19 spreads rapidly and has a higher prevalence in cancer patients around the globe, it is imperative to define the risk factors, outcomes, and features of this group. To accomplish this, in Pakistan, the Ministry of Health formulated a registry of patients nationwide who had COVID-19 in the earliest days of the pandemic. All cases of COVID-19 confirmed by positive polymerase chain reaction (PCR) were registered and managed by the physicians who attended them. The data used in our study, including clinical, demographic, and laboratory data of patients who presented with solid malignancy and positive COVID-19 confirmed by PCR, were collected from the national registry of COVID19 patients in Pakistan.

\section{Materials And Methods}

On February 26, 2020, the first case of COVID-19 was reported in Pakistan, and the Ministry of Health responded swiftly by devising a national COVID-19 registry. This was established to develop a database to monitor cases, testing, and strategies to devise a treatment for the disease. The prospective registration of COVID-19 cases in all private and government hospitals was conducted, and cases that had been previously diagnosed were added to the registry. This form-based registry is ongoing, and attending clinicians are updating the database electronically on a periodic basis.

The data collected after identification have been made available to investigators for further analysis. All the patients included in this data were accurately diagnosed with confirmed PCR analysis. The samples were obtained via nasal swabs. We did not include patients who presented with negative PCR results, but they were recorded in the registry.

The timeline of this study was between April and September 2020. It was conducted at the Dow University of Health Sciences after ethical approval from the Institutional Review Board, and the informed consent requirement was waived. The study included adult patients ( $\geqslant 18$ years) with solid tumors who were confirmed COVID-19 cases. For analysis of solid tumors, codes from the International Classification of Diseases, 10th edition were used. Patients who presented with in situ carcinomas, as well as those with malignancies related to hematology, were excluded from the study.

We assessed features including demographic treatment information for cancer and COVID-19. The assessment of laboratory tests included coagulation tests, CT scans, biochemistry serum tests, and blood counts. The reference values of the corresponding centers were used to categorize laboratory results to consider normal and abnormal reference values. The CT scans were classified as "normal," "consistent with viral pneumonia," or "other findings." We did not use a predefined criterion to classify CT scans; it was solely at the discretion of the local hospital radiologist. There was no CT scan central review. To extract the information on cancer treatment, Anatomical Therapeutic Chemical codes were used from the existing database. We defined "receiving active cancer treatment" as patients being treated for cancer within four weeks of diagnosis of COVID-19. Details of COVID-19 treatment, including azithromycin, remdesivir, and hydroxychloroquine (HCQ), and information regarding intensive care unit (ICU) admission were collected. The follow-up period was defined from the first confirmed PCR test date until 30 days after this test. The patients included in the study were those who were followed up for at least 30 days and those who died within 30 days of continuing COVID-19. The final outcome of the study was 30-day mortality.

\section{Statistical analysis}

The mean \pm standard deviation (SD) was used to characterize the baseline clinical and demographic variables and frequencies for categorization. To determine comorbidities, sex, age, lab parameters, and the treatment methods correlated with 30-day mortality, univariate analysis, chi-square test, and Student's t-test were used. To determine the relevant clinical factors correlated with mortality within 30 days, multivariate analysis using logistic regression was used. Certain variables that showed significant correlation levels of $\alpha$ $=0.020$ were included in the multivariate model. Data completeness and clinical validation were also included. A backward selection was used in the logistic regression analysis in the multivariate model. To assess the model fit, Hosmer-Lemeshow goodness-of-fit statistics were used. For statistically significant influence, the type 1 error level, which was less than 5\%, was used. The Statistical Package for the Social Sciences version 24 (IBM Corp., Armonk, NY) was employed to perform all statistical analyses.

\section{Results}

The total number of participants was 1,519. The age, gender, demography, and clinical and treatment characteristics of the patients are shown in Table 1. The participants' ages were in the range of 20-95 years, and the median age was 62 years. Patients older than 65 years made up $38.8 \%$ of the sample, and 750 were men. Of the patients in our study population, $3.15 \%$ had prostate cancer, $10.20 \%$ had colorectal cancer, $2.76 \%$ had breast cancer, and $10.46 \%$ had lung cancer. Moreover, $25.93 \%$ of the patients presented with at least one comorbidity. The most common comorbid disease was hypertension at $25.7 \%$, followed by diabetes mellitus (12.7\%) and chronic obstructive pulmonary disease (6.84\%). The least common was kidney disease at $4.73 \%$. Clinical features correlated with high mortality risk were hypertension, chronic liver disease, diabetes, and chronic kidney disease, which were found in older men. The university centers' mortality rate 


\section{Cureus}

was $9.7 \%$, and the public hospital mortality rate was $3.9 \%(\mathrm{p}=0.002)$. Overall, $25 \%$ of the patients had received systemic anti-cancer therapy within four weeks before the diagnosis of COVID-19; in this context, $13.5 \%$ received cytotoxic therapy, $11.1 \%$ received hormone therapy, and the remaining $3.6 \%$ received targeted therapy. Immunotherapy was administered to only two patients.

\begin{tabular}{|c|c|c|c|c|}
\hline Variables & $\mathbf{N}$ & Deceased & Surviving & P-value \\
\hline Total patients & 1,519 & $72(4.74 \%)$ & $1,447(95.26 \%)$ & \\
\hline Age of the patients (median and min-max) & $62(20-95)$ & $68(43-86)$ & $62(20-95)$ & $<0.001>$ \\
\hline \multicolumn{5}{|l|}{ Gender } \\
\hline Female & $769(50.6 \%)$ & $19(2.5 \%)$ & $753(97.5 \%)$ & \\
\hline Male & $750(49.37 \%)$ & $53(7.06 \%)$ & $694(92.3 \%)$ & $<0.001>$ \\
\hline \multicolumn{5}{|l|}{ Age groups } \\
\hline$\leq 35$ & $73(4.80 \%)$ & $1(1.38 \%)$ & $71(97.26 \%)$ & $<0.001>$ \\
\hline $36-50$ & $274(18.03 \%)$ & $5(1.82 \%)$ & $269(98.17 \%)$ & \\
\hline $51-65$ & $589(38.8 \%)$ & $23(3.90 \%)$ & $565(95.92 \%)$ & \\
\hline$>65$ & $583(38.39 \%)$ & $42(7.20 \%)$ & $541(92.79 \%)$ & \\
\hline \multicolumn{5}{|l|}{ Hospital type } \\
\hline Private & $279(18.3 \%)$ & $18(6.5 \%)$ & $261(93.5 \%)$ & \\
\hline Public & $1,058(69.5 \%)$ & $41(3.9 \%)$ & $1,017(96.1 \%)$ & 0.002 \\
\hline University & $179(11.78 \%)$ & $18(9.7 \%)$ & $161(89.94 \%)$ & \\
\hline \multicolumn{5}{|l|}{ Comorbidities } \\
\hline Diabetes & $194(12.7 \%)$ & $19(9.8 \%)$ & 175 (90.2\%) & 0.004 \\
\hline Hypertension & $391(25.7 \%)$ & $28(7.2 \%)$ & $363(92.8 \%)$ & 0.032 \\
\hline Chronic kidney disease & $71(4.73 \%)$ & $6(8.33 \%)$ & $64(88.9 \%)$ & 0.025 \\
\hline Chronic obstructive pulmonary disease & $104(6.84 \%)$ & $10(9.61 \%)$ & $94(88.7 \%)$ & 0.009 \\
\hline Chronic liver disease & $31(2.04 \%)$ & 7 (22.58\%) & $24(77.41 \%)$ & $<0.001>$ \\
\hline Cerebrovascular event & $69(4.54 \%)$ & $4(5.8 \%)$ & $65(94.2 \%)$ & 0.77 \\
\hline \multicolumn{5}{|l|}{ Number of comorbidities } \\
\hline 0 & $530(34.89 \%)$ & $19(3.58 \%)$ & $511(96.8 \%)$ & $<0.001>$ \\
\hline 1 & $394(25.93 \%)$ & $17(4.31 \%)$ & $377(95.6 \%)$ & \\
\hline 2 & $295(19.42 \%)$ & $6(2.0 \%)$ & $289(98.0 \%)$ & \\
\hline$\geq 3$ & $300(19.74 \%)$ & $30(10 \%)$ & $270(90 \%)$ & \\
\hline \multicolumn{5}{|l|}{ Cancer type } \\
\hline Lung & $159(10.46 \%)$ & $7(4.40 \%)$ & $152(95.59 \%)$ & $<0.001>$ \\
\hline Colorectal & $155(10.20 \%)$ & $17(10.96 \%)$ & $138(89.03 \%)$ & \\
\hline Breast & $42(2.76 \%)$ & $3(7.14 \%)$ & $39(92.85 \%)$ & \\
\hline Skin & $312(20.53 \%)$ & $6(19.23 \%)$ & $306(98.07 \%)$ & \\
\hline Renal & $165(10.86 \%)$ & $9(5.45 \%)$ & $156(94.54 \%)$ & \\
\hline Prostate & $48(3.15 \%)$ & $3(6.25 \%)$ & $45(93.75 \%)$ & \\
\hline Other & $130(8.55 \%)$ & 10 (7.69\%) & $120(92.31 \%)$ & \\
\hline Thyroid and endocrine glands & 144 (9.47\%) & $4(2.77 \%)$ & $140(97.3 \%)$ & \\
\hline
\end{tabular}




\section{Cureus}

$\begin{array}{lccr}\text { Bladder } & 364(23.96 \%) & 34(9.34 \%) & 330(90.65 \%) \\ \text { Targeted treatment }^{\mathrm{a}} & 55(3.62 \%) & 5(9.1 \%) & 50(90.9 \%) \\ \text { Cytotoxic treatment }^{\mathrm{a}} & 205(13.5 \%) & 20(9.8 \%) & 185(90.2 \%) \\ \text { Hormonal treatment }^{\mathrm{a}} & 169(11.1 \%) & 6(3.6 \%) & 163(96.4 \%)\end{array}$

TABLE 1: Analysis of age, gender, demographic features, clinical features, and treatment.

aTreatment within four weeks of coronavirus disease 2019 diagnosis.

In total, $58 \%$ of patients underwent CT scans at least once. In $95.1 \%$ of patients, CT scan findings of the thorax were consistent with viral pneumonia. In the first PCR test, $87.6 \%$ of the patients presented with a confirmed COVID-19 diagnosis. The proportion increased to $97 \%$ in the second PCR test, and in the third test, the proportion reached 99.3\%. Patients who presented with a negative PCR test in the first attempt showed a higher mortality rate compared with the positive ones $(12.2 \%$ vs. $4.0 \%$; $\mathrm{p}<0.0001)$. Table 2 shows the laboratory results of positive cancer patients diagnosed with COVID-19. 


\section{Cureus}

\begin{tabular}{|c|c|c|c|c|c|}
\hline Variables & $\mathbf{N}$ & Values & Deceased & Surviving & P-value \\
\hline Leukocyte $\left(\times 10^{3} / \mu \mathrm{L}\right)$ & 1,042 & $5.60(4.2-7.5)$ & $7(4.9-13.4)$ & $5.6(4.3-7.5)$ & 0.001 \\
\hline $\mathrm{Hb}(\mathrm{g} / \mathrm{dL})$ & 1,059 & $12.6 \pm 1.99$ & $10.9 \pm 2.5$ & $12.8 \pm 2.01$ & $<0.001>$ \\
\hline Neutrophil $\left(\times 10^{3} / \mu \mathrm{L}\right)$ & 842 & $3.7(2.7-5.6)$ & $6.4(4.1-13.3)$ & $3.6(2.6-5.2)$ & $<0.001>$ \\
\hline Lymphocyte $\left(\times 10^{3} / \mu \mathrm{L}\right)$ & 1,030 & $1.2(0.8-1.7)$ & $0.8(0.4-1.3)$ & $1.2(0.8-1.7)$ & $<0.001>$ \\
\hline Neutrophil-Iymphocyte ratio $>4$ & 831 & $4(1.9-5.02)$ & $8(4.5-14.92)$ & $3.01(1.9-4.3)$ & $<0.001>$ \\
\hline Platelet $\left(\times 10^{3} / \mu \mathrm{L}\right)$ & 1,048 & $192(152-247)$ & $172(108-258)$ & $193(154-247)$ & 0.073 \\
\hline Fibrinogen (mg/dL) & 209 & $439 \pm 151$ & $481 \pm 249$ & $433 \pm 134$ & 0.38 \\
\hline C-reactive protein (mg/L) & 989 & $23.01(7.7-75.8)$ & $110(60.1-200)$ & $21.02(7.4-64.3)$ & $<0.001>$ \\
\hline D-dimer $(\mu \mathrm{g} / \mathrm{L})$ & 536 & $710(385-1,329)$ & $1,952(1,048-4,989)$ & $654(380-1,095)$ & $<0.001>$ \\
\hline Ferritin (ng/L) & 390 & $188(80.2-405)$ & $761(155-1,310)$ & $178(79.1-390)$ & $<0.001>$ \\
\hline Procalcitonin (ng/mL) & 340 & $0.2(0-0.3)$ & $0.3(0.2-1.1)$ & $0(1-1.2)$ & $<0.001>$ \\
\hline International normalized ratio & 439 & $2(1.8-2.0)$ & $1.0(1.10-1.80)$ & $1(1.5-1.8)$ & 0.001 \\
\hline Iroponın-I (mg/L) & 231 & $3.5(0-8)$ & $11.4(5.2-31.1)$ & $2.5(0-5.2)$ & $<0.001>$ \\
\hline Lactate dehydrogenase (U/L) & 639 & $242(189-325)$ & $385(245-652)$ & $242(132-318)$ & $<0.001>$ \\
\hline Bilirubin (mg/dL) & 592 & $0.5(0.4-0.8)$ & $0.7(0.5-1.2)$ & $0.3(0.2-0.5)$ & 0.001 \\
\hline Albumin (g/dL) & 500 & $3.10 \pm 0.4$ & $3.1 \pm 0.4$ & $3.8 \pm 0.6$ & $<0.001>$ \\
\hline Alanine aminotransferase (U/L) & 859 & $22(15-30)$ & $21(11-34)$ & $22(13-29)$ & 0.68 \\
\hline Aspartate aminotransterase (U/L) & 910 & $27.01(20-36.5)$ & $35(21-63)$ & $24(20-34)$ & 0.005 \\
\hline Alkaline phosphatase (U/L) & 451 & $80(60-110)$ & $97.1(81.1-150.1)$ & $77(59-100)$ & $<0.001>$ \\
\hline Gamma-glutamyl transterase (U/L) & 480 & $31(20.18-55.4)$ & $57(33-10.1)$ & $31(20-58)$ & $<0.001>$ \\
\hline Sodium (mEq/L) & 510 & $137 \pm 6$ & $134 \pm 8$ & $139 \pm 2$ & 0.80 \\
\hline Creatinine (mg/dL) & 851 & $0.9(0.8-1.2)$ & $0.8(0.6-1.4)$ & $0.6(0.5-0.9)$ & 0.036 \\
\hline Creatine phosphokinase (U/L) & 445 & $73(46-120)$ & $69.10(34-118.4)$ & $74(48-120)$ & 0.74 \\
\hline
\end{tabular}

\section{TABLE 2: Analysis of laboratory results.}

$\mathrm{Hb}$ : hemoglobin

In $73 \%$ of patients, specified COVID-19 therapy was performed, and the most common treatments were HCQ plus azithromycin in $45.16 \%$ and total HCQ in $69.7 \%$ of patients. Total favipiravir was given to $20.4 \%$ of patients to salvage refractory cases. This treatment was also used as first-line therapy for severe pneumonia patients and ICU patients. Since the outbreak of COVID-19 occurred during the influenza season, oseltamivir was employed in $51 \%$ of patients. The median follow-up time for our cohort was 15 days, with a minimum of one and a maximum of 74 days. In the study, $56.6 \%$ of patients were hospitalized and $7.1 \%$ of them died within 30 days. Among hospitalized patients, $11.32 \%$ were admitted to the ICU and $30.8 \%$ of them died; invasive mechanical ventilation was given to $7.4 \%$ of patients and $39.8 \%$ of them died. This analysis of treatment regimens and patient outcomes is shown in Table 3. 


\section{Cureus}

\begin{tabular}{|c|c|c|c|c|}
\hline Treatment regimens & $\mathbf{N}$ & Deceased & Surviving & P-value \\
\hline Total azithromycin & $701(45.8 \%)$ & 39 (5.6\%) & $662(94.4 \%)$ & 0.34 \\
\hline Total HCQ & $1,066(69.7 \%)$ & $59(5.5 \%)$ & $1,007(94.5 \%)$ & 0.25 \\
\hline Total lopinavir & $46(3.0 \%)$ & $4(8.7 \%)$ & $42(91.3 \%)$ & 0.28 \\
\hline Iotal tavipiravir & $311(20.4 \%)$ & $4 /(15.1 \%)$ & $264(84.9 \%)$ & $<0.001\rangle$ \\
\hline Azithromycin + $\mathrm{HCQ}+$ favipiravir & $218(14.35 \%)$ & $28(16.27 \%)$ & $190(87.15 \%)$ & $<0.001\rangle$ \\
\hline HCQ + azithromycin & $687(45.1 \%)$ & $39(5.7 \%)$ & $648(94.3 \%)$ & 0.34 \\
\hline Supportive therapy alone & $406(26.7 \%)$ & $10(2.5 \%)$ & 396 (97.5\%) & 0.005 \\
\hline Hospitalization & $862(56.6 \%)$ & $61(7.1 \%)$ & $801(92.9 \%)$ & $<0.001>$ \\
\hline ICU admission & $172(11.32 \%)$ & $53(30.8 \%)$ & $119(69.1 \%)$ & $\langle 0.001\rangle$ \\
\hline Intubation & $113(7.4 \%)$ & $45(39.8 \%)$ & $68(60.2 \%)$ & $<0.00$ \\
\hline
\end{tabular}

TABLE 3: COVID-19 treatment regimens and patient outcomes.

HCQ: hydroxychloroquine; ICU: intensive care unit; COVID-19: coronavirus disease 2019

In a subset of data, we ran the multivariate model and included demographic and clinical information that was available at the time, including age, cancer type, diabetes, chemotherapy, and other comorbidities. The analysis is shown in Table 4. 


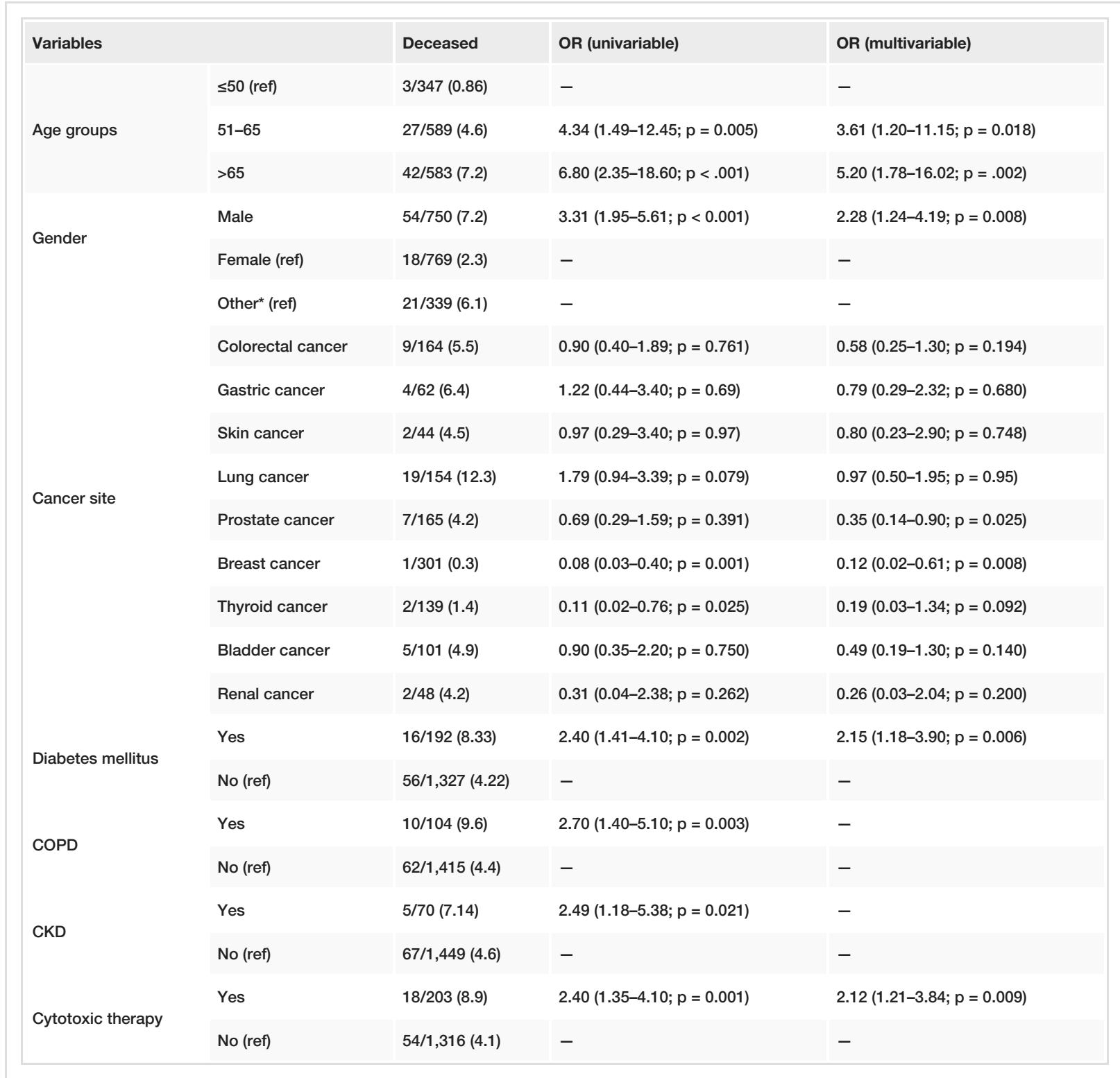

TABLE 4: Univariate and multivariate regression analysis of potential baseline clinical variables.

OR: odds ratio; COPD: chronic obstructive pulmonary disease; CKD: chronic kidney disease

\section{Discussion}

Mortality in COVID-19 patients within the first 30-day period was found to be $4.74 \%$ in this study of adult patients with solid tumors. Lung cancer patients were found to be at a higher risk of mortality, whereas prostate cancer, thyroid cancer, and breast cancer patients showed lower mortality risk. Cytotoxic treatment, diabetes, old age, and time since cancer diagnosis were found to be crucial predictive factors of increased mortality risk. Lymphopenia was found to be a high mortality risk factor in laboratory outcomes, whereas the use of azithromycin and HCQ was found to have no effect on the 30-day mortality timeline. Compared with noncancer patients, the mortality rate of cancer patients was found to be higher in COVID19 cases. The first report from China reported the case fatality rate (CFR) to be $28.6 \%$. Two subsequent reports from China showed that the CFR was $20 \%$. Solid tumor mortality of $12 \%$ and hematological malignancy of $14 \%$ were found in a study done by the COVID-19 and Cancer Consortium (CCC19). One study from the Memorial Sloan Kettering Cancer Center (MSKCC) reported the CFR as 12\% [9-13].

ICU support was found to be high in Pakistan because the rate of patients who died from COVID-19 without admission to an ICU was lower. As the COVID-19 outbreak hit Pakistan later than it did China and Europe, ICUs were made available in higher numbers in Pakistan to comply with COVID-19 readiness. Seventy-one percent of patients who died underwent either invasive or noninvasive ventilation; this figure is found to be higher than those reported from the MSKCC, the CCC19, and the United Kingdom Coronavirus Cancer 
Monitoring Project series [13-15]. Higher availability of ventilation and intensive care can also be the reason for a lower mortality rate in Pakistan for COVID-19. As COVID-19 cancer patients were clustered nationwide, intensive filiation studies may also have contributed to lower COVID-19 severity in the study [16].

Cytotoxic treatment for weeks after the diagnosis of COVID-19 turned out to represent a high risk of mortality compared with endocrine therapy and targeted therapy. Some studies have contradicted these results in that they did not show a correlation between higher mortality rates and cytotoxic therapy. However, a study showed a positive correlation between recent cytotoxic therapy and the mortality rate in COVID-19 patients. It also presented a higher risk in patients who received cytotoxic treatment within two weeks, but if the time was more than three weeks, the risk was lower [17]. From the results of our study, we can conclude that during cytotoxic treatment, a pandemic patient who can move to alternative treatment should be shifted to safer therapies, and a patient who has no alternative treatment option should continue with cytotoxic treatment with the utmost care and strong protective measures against COVID-19. An unfavorable prognosis was presented in the MSKCC study for COVID-19 patients who received or were receiving immunotherapy [15]. In our study, we only had two patients who received immunotherapy, and this issue needs further analysis.

Nearly $50 \%$ of patients in our study group received azithromycin and HCQ treatment; this treatment is widely adopted and endorsed by the Ministry of Health in Pakistan for patients showing clear symptoms of COVID-19. A number of previous studies have shown that patients who receive HCQ treatment are at a high risk of mortality $[18,19]$. However, the results of our study differ from these studies because we could not find an increased risk of mortality in patients who were treated with HCQ with or without azithromycin compared with patients who did not receive these treatments. These results may be because patients underwent HCQ treatment as a consequence of higher risk factors and symptoms. As we used registry-based data in our study, it is safe to say that some of the risk factors were incomplete because of multiple assessments; hence, we did not compare our study with formal COVID-19 treatments.

A higher mortality rate is also correlated with higher international normalized ratio and D-dimer levels, suggesting coagulopathy. The poor prognosis of this disease is also correlated with gamma-glutamyl transferase, troponin I, alkaline phosphate, and creatinine. Consideration of these factors can be useful in the prognosis of early screening of critical disease, especially when determining inflammation dynamics and phases of immunity and early treatment $[20,21]$. However, these biomarkers are not available for each patient, so we did not use a single marker or a group of markers for our prognostic measures. Our study suggests that these biomarkers can be helpful in further studies.

The major limitation of our study was missing information on COVID-19 patients; we only included the PCR test, but the true number of COVID-19 patients may be higher. Moreover, a long-term follow-up of more than 30 days may compile better results on mortality in cancer patients as a result of COVID-19.

\section{Conclusions}

COVID-19 has a higher prevalence in cancer patients than in the general population, resulting in higher mortality rates. In our study, we focused on solid tumor patients infected with COVID-19, and this is the largest series involving such patients to date. The results confirm previous reports' clinical features and the higher mortality rate in this group of patients; however, the mortality rate was lower than that in China, as well as Western and European countries. Our study showed that clinical readiness and supportive intensive care can decrease mortality rates in COVID-19 and cancer patients, as well as patients with other lifethreatening diseases. However, it is imperative to better identify treatment regimens and strategies from new interventional and observational studies of larger groups.

\section{Additional Information}

\section{Disclosures}

Human subjects: Consent was obtained or waived by all participants in this study. Dow University of Health Sciences issued approval IRB-1805/DUHS/Approval/2020/2597. Animal subjects: All authors have confirmed that this study did not involve animal subjects or tissue. Conflicts of interest: In compliance with the ICMJE uniform disclosure form, all authors declare the following: Payment/services info: All authors have declared that no financial support was received from any organization for the submitted work. Financial relationships: All authors have declared that they have no financial relationships at present or within the previous three years with any organizations that might have an interest in the submitted work. Other relationships: All authors have declared that there are no other relationships or activities that could appear to have influenced the submitted work.

\section{References}

1. Hassan SA, Sheikh FN, Jamal S, Ezeh JK, Akhtar A: Coronavirus (COVID-19): a review of clinical features, diagnosis, and treatment. Cureus. 2020, 12:e7355. 10.7759/cureus.7355

2. Pisters KM, Newman RA, Coldman B, et al.: Phase I trial of oral green tea extract in adult patients with solid 
tumors. J Clin Oncol. 2001, 19:1830-8. 10.1200/JCO.2001.19.6.1830

3. de Azambuja E, Brandão M, Wildiers H, et al.: Impact of solid cancer on in-hospital mortality overall and among different subgroups of patients with COVID-19: a nationwide, population-based analysis. ESMO Open. 2020, 5:e000947. 10.1136/esmoopen-2020-000947

4. Fuentes-Antrás J, Manzano A, Marquina G, et al.: A snapshot of COVID-19 infection in patients with solid tumors. Int J Cancer. 2020, 148:2389-97. 10.1002/ijc.33420

5. Tang LV, Hu Y: Poor clinical outcomes for patients with cancer during the COVID-19 pandemic . Lancet Oncol. 2020, 21:862-4. 10.1016/S1470-2045(20)30311-9

6. Razanamahery J, Soumagne T, Humbert S, et al.: Does type of immunosupression influence the course of Covid-19 infection?. J Infect. 2020, 81:e132-5. 10.1016/j.jinf.2020.05.075

7. Zhang H, Han H, He T, et al.: Clinical characteristics and outcomes of COVID-19-infected cancer patients: a systematic review and meta-analysis. J Natl Cancer Inst. 2021, 113:371-80. 10.1093/jnci/djaa168

8. Meng Y, Lu W, Guo E, et al.: Cancer history is an independent risk factor for mortality in hospitalized COVID-19 patients: a propensity score-matched analysis. J Hematol Oncol. 2020, 13:75. 10.1186/s13045020-00907-0

9. Robilotti EV, Babady NE, Mead PA, et al.: Determinants of COVID-19 disease severity in patients with cancer. Nat Med. 2020, 26:1218-23. 10.1038/s41591-020-0979-0

10. Liu C, Li L, Song K, et al.: A nomogram for predicting mortality in patients with COVID-19 and solid tumors: a multicenter retrospective cohort study. J Immunother Cancer. 2020, 8:e001314. 10.1136/jitc-2020001314

11. Mehta V, Goel S, Kabarriti R, et al.: Case fatality rate of cancer patients with COVID-19 in a New York hospital system. Cancer Discov. 2020, 10:935-41. 10.1158/2159-8290.CD-20-0516

12. Al-Quteimat OM, Amer AM: The impact of the COVID-19 pandemic on cancer patients . Am J Clin Oncol. 2020, 43:452-5. 10.1097/COC.0000000000000712

13. Kuderer NM, Choueiri TK, Shah DP, et al.: Clinical impact of COVID-19 on patients with cancer (CCC19): a cohort study. Lancet. 2020, 395:1907-18. 10.1016/S0140-6736(20)31187-9

14. Sud A, Jones ME, Broggio J, et al.: Collateral damage: the impact on outcomes from cancer surgery of the COVID-19 pandemic. Ann Oncol. 2020, 31:1065-74. 10.1016/j.annonc.2020.05.009

15. Luo J, Rizvi H, Egger JV, Preeshagul IR, Wolchok JD, Hellmann MD: Impact of PD-1 blockade on severity of COVID-19 in patients with lung cancers. Cancer Discov. 2020, 10:1121-8. 10.1158/2159-8290.CD-20-0596

16. Wysocki PJ, Kwinta $€$, Potocki P, et al.: Systemic treatment of patients with solid tumors during the COVID19 (SARS-CoV-2) pandemic-comprehensive recommendations of the Polish Society of clinical oncology. Oncol Clin Pract. 2020, 16:41-51. 10.5603/OCP.2020.0012

17. Marté JL, Toney NJ, Cordes L, Schlom J, Donahue RN, Gulley JL: Early changes in immune cell subsets with corticosteroids in patients with solid tumors: implications for COVID-19 management. J Immunother Cancer. 2020, 8:e001019. 10.1136/jitc-2020-001019

18. Bogani G, Ditto A, Bosio S, Brusadelli C, Raspagliesi F: Cancer patients affected by COVID-19: experience from Milan, Lombardy. Gynecol Oncol. 2020, 158:262-5. 10.1016/j.ygyno.2020.06.161

19. Lee LYW, Cazier JB, Starkey T, et al.: COVID-19 prevalence and mortality in patients with cancer and the effect of primary tumour subtype and patient demographics: a prospective cohort study. Lancet Oncol. 2020, 21:1309-16. 10.1016/S1470-2045(20)30442-3

20. Schrag D, Hershman DL, Basch E: Oncology practice during the COVID-19 pandemic . JAMA. 2020, 323:2005-6. 10.1001/jama.2020.6236

21. He W, Chen L, Chen L, et al.: COVID-19 in persons with haematological cancers . Leukemia. 2020, 34:163745. 10.1038/s41375-020-0836-7 